\title{
Stanistaw Russocki
}

\section{Gesellschaft und Ständestaat im Polen des ausgehenden Mittelalters}

\author{
Einige strittige Probleme
}

Die bisherige Historiographie nimmt übereinstimmend an, daß im 14.-15. Jahrhundert in Polen sowohl eine standesmäßig organisierte Gesellschaft sowie auch Formen des politischen Systems, das Ständemonarchie genannt wird - d.h. einer Monarchie, in deren Rahmen die privilegierten Stände einen beständigen und institutionalisierten Einfluß auf die wichtigsten politischen Entscheidungen errungen hatten -, existierten. Umstritten sind bei den einzelnen Forschern einzig und allein die Zeitbegrenzungen, innerhalb derer die erwähnten Erscheinungen auftreten. Es besteht auch ein allgemeiner Konsens darüber, daß die im Laufe des 16. Jahrhunderts eingebürgerte, für Polen spezifische Regierungsform, die als Adelsrepublik bekannt ist, in ihrem Wesen ein Ständestaat war, mit der Einschränkung, daß er durch einen einzigen, privilegierten Stand - die Schlachta - dominiert wurde ${ }^{1}$.

Erst in der letzten Zeit wurde von H. Samsonowicz die Stichhaltigkeit einer derartigen Auffassung in Frage gestellt. Seiner Meinung nach spiegeln die staatlichen normativen Texte und allgemeinen Privilegien des 14. bis 15. Jahrhunderts eine gänzlich andere Wirklichkeit wider als die allgemein angenommene, die in der Analyse zahlreicher Akten der Land- oder Stadtgerichte zutage tritt. Die erstgenannte Quellengattung programmierte sozusagen von oben die Einteilung der Gesellschaft in Stände. Aber bei der Lektüre der Gerichtsakten springt nicht nur eine ungefestigte Begrifflichkeit ins Auge, die dieselben Personen verschiedenartigen Ständekategorien zuweist, sondern auch ein weitgehendes Schwanken, wenn es sich um die Barrieren zwischen den Gruppen handelt, um zahlreiche Verbindungen der Bürger mit den Adeligen und Bauern, und zwar nicht nur ökonomischer Natur, sondern auch etwa im Bereich der Eheschließungen. Demgemäß stellt der genannte Forscher fest, daß die polnische Gesellschaft ihren Ständecharakter erst im 16. Jahrhundert angenommen hat, als sich

1 Da die in den dargelegten Betrachtungen besprochenen Fakten hinlänglich bekannt sind, erübrigt sich für den Verfasser ihre Dokumentierung. Sie sind - freilich etwas veraltet - auch in den vielen Verallgemeinerungen der ausführlichen Synthese 2 . Wojciechowskis, L'Etat polonais au Moyen âge (Paris 1949) enthalten. Die Anmerkungen sind auf die Fälle begrenzt, wo bestimmte Ansichten erläutert oder neuere Erkenntnisse einzelner, in Fremdsprachen publizierter Probleme dargelegt werden müssen. 
die Übermacht der Schlachta über das Bürgertum und das Bauerntum gefestigt hatte ${ }^{2}$.

Ohne Antwort läßt H. Samsonowicz die Frage nach der Natur der früheren gesellschaftlichen Gliederungen. Deswegen nimmt der Referent - eingedenk der Rolle, die möglichst eindeutige allgemeine Begriffskategorien bei vergleichenden Forschungen spielen - die Polemik unter dem eben dargestellten Blickpunkt auf, wobei er als Ausgangspunkt die allgemeinen Beobachtungen nimmt, die die vergleichende Analyse der europäischen Ständestrukturen bietet.

Einleitend sei gesagt, daß $\mathrm{H}$. Samsonowicz wohl kaum Recht hat, wenn er den Vorgängern Willkür bei der Verwendung des Terminus „Stand“ vorwirft ${ }^{3}$. Unserer Meinung nach hatte die frühere Literatur, die unabhängig von konkreten Formulierungen über Stände schrieb, immer Gruppen von Personen im Auge, die sich durch die im Rahmen der staatlichen Organisation ausgeübten Funktionen voneinander unterschieden und aus diesem Grunde mit einem besonderen rechtlichen Status ausgestattet waren. Dagegen kann man vielen bisherigen polnischen Auffassungen vorwerfen, daß ihnen eine vertiefte Reflexion über die in Zeit und Ort veränderlichen Funktionen der gesellschaftlichen Gliederungen fehlt, die in den Kategorien von Ständen ausgedrückt werden ${ }^{4}$.

Die funktionsgemäße Einteilung der Gesellschaft in Gruppen war schon den Indoeuropäern bekannt. Sie drückte das aus, was die derzeitige Soziologie „Distribution gesellschaftlicher Rollen“" nennt. Diese Einstellung vererbte sich auf das christliche Europa, wobei dieses jene theoretischen Grundlagen als Lehre von den Ständen ordines - ausbildete. Dieser Terminus wurde verschiedenen Gruppen von Personen beigelegt, immer jedoch jenen, die sich in irgendeiner Weise durch eine gemeinsame Position innerhalb einer gegebenen Gemeinschaft auszeichneten, zum Beispiel den Geistlichen innerhalb der Weltlichen, den Verheirateten oder Witwern inmitten der Leute von freiem Stand. Zu Beginn bestanden drei ordines (oratores, bellatores, laboratores), deren Zahl sich mit der Zeit vergrößerte. Als Unterscheidungskriterien dienten bei solchen Einteilungen sowohl die ausgeübte Funktion als auch der damit einhergehende Status, der sich durch einen Komplex von Rechten und Pflichten ausdrückte.

Gewiß war dies eine Blickrichtung, die die gesellschaftliche Wirklichkeit von oben und programmierend betrachtete, was für die damaligen Zentren sowohl geistlicher wie auch weltlicher Macht charakteristisch war. Wenn wir aber den Blick von unten nach oben richten, können wir auch bei den Personen, die eine bestimmte Position einnahmen, das Entstehen horizontaler Verknüpfungen auf der Grundlage von Solidarität und dem Bewußtsein der gemeinsamen Lage beobachten. Diese Bande wurden dann besonders fest, wenn es um die Notwendigkeit des Kampfes zum Schutz einmal

\footnotetext{
${ }^{2}$ Cf. Henryk Samsonowicz, Stände und zwischenständische Beziehungen in Polen im 15. Jahrhundert, in: Jahrbuch für Geschichte 23 (Berlin, 1981) $103 \mathrm{ff}$.

${ }^{3}$ Ebd., 104.

${ }^{4}$ Cf. Stanistaw Russacki, Die mittelalterlichen Stände als Kategorie der Gesellschaftsschichtung, in: Acta Poloniae Historica (weiter zit. APH) XLVIII (1983) $5 \mathrm{ff}$. Im vorliegenden Text rekurriert der Verfasser auf frühere Feststellungen und präzisiert seine Position in gewissen Punkten genauer.
} 
erworbener Rechte oder um deren Ausweitung ging. Auf diese Weise entstand das Kollektivbewußtsein der Zugehörigkeit zu einem bestimmten, korporativ organisierten Stand, das um so stärker und dauerhafter war, je größer die gesellschaftlich-politische Position war, die die betreffende Gruppe einnahm, und je mehr diese sich von der übrigen Gesellschaft distanzieren und abgrenzen wollte. Es ist also nicht verwunderlich, daß sich im Rahmen der einzelnen Königreiche oder Fürstentümer der geistliche und der Adelsstand (bisweilen der Herrenstand und der Adelsstand) am schnellsten und am vollständigsten herauskristallisierten.

Die Tatsache, daß die einzelnen Schichten oder Gruppen sich in Stände abgrenzten, schließt keineswegs aus, daß innere Einteilungen und Antagonismen von gesellschaftlich-wirtschaftlichem Charakter nicht fortbestanden hätten. Ebenso schließen die Standesstrukturen nicht aus, daß sich eine gesellschaftliche Mobilität bemerkbar machte, daß die einen höhere Positionen in der Hierarchie, andere wiederum niedrigere einnehmen wollten oder mußten. Seit langem wurde zutreffend festgestellt, daß die mittelalterlichen Stände keine Kasten waren ${ }^{5}$.

Wie es scheint, wird die Tatsache, daß eine gewisse Gesellschaft eine Ständegesellschaft war, nicht nur durch ihre ideale Segmentierung bestimmt - eine solche wurde niemals erreicht -, sondern durch das Streben nach einer der Ständekonzeption gemäßen Strukturierung. Dieses Streben bestand sowohl aus den Maßnahmen der politischen Behörden wie aus den Bemühungen der gesellschaftlichen Gruppen, die sich der Tatsache gemeinsamer Leistungsfähigkeit bewußt waren und sie in ihrem Wirken ausnützten. Als Phänomen der erwähnten Tendenz muß auch der Prozeß der Differenzierung innerhalb der Stände angesehen werden ${ }^{6}$, zum Beispiel das Bestreben der Gruppen des Patriziates, sich vom gemeinen Volk zu isolieren.

Schematisierung und Verfestigung des Bildes der Ständestrukturen wurden durch die laufende Politik der Staaten gefördert. Bei der Regulierung der Pflichten der einzelnen Schichten und Gruppen ihrer Untertanen bedienten sie sich einer vereinfachten globalen Vision von bestehenden Systemen, ohne die besonderen Situationen in Betracht zu ziehen. Auf ähnliche Weise geht ja auch der heutige Staat vor, obwohl er soviel reicher an verschiedenartigen Wirkungs- und $Z$ wangsmitteln ist.

Da gerade die Rede vom Staat ist, scheint es sinnvoll zu sein, einen begrifflichen Unterschied zwischen jenen Ständen einzuführen, die als eine Form der Organisation gesellschaftlicher Differenzierung des Ancien Régime existierten, und denen, die politische Stände darstellten. Zu den letzteren zählen wir nur diejenigen Ständegruppen, denen es gelungen ist, persönlich oder mit Hilfe von Vertretern, an der Ausübung der staatlichen Oberhoheit teilzuhaben.

${ }^{5}$ Cf. E. Lousse, Les ordres d'Ancien régime n'étaient pas des castes, in: IX Congrès International des Sciences Historiques Paris 1950. Université de Louvain. Recueil de travaux d'histoire et de philologie, 3-e série 45-e fasc. (Louvain 1952) $253 \mathrm{ff}$; Roland Mousnier, Les hierarchies sociales de 1450 à nos jours (Paris 1969).

${ }^{6}$ Cf. K. Modzelewski, L'organizzazione dello stato polacco nel secoli X-XIII. La società e le strutture del potere, in: Settimane di Studio del Centro Italiano di Studi sull'Alto Medioevo XXX, Gli Slavi ... (Spoleto 1983) 557 ff., idem, Le système du ,ius ducale“ en Pologne et le concept du féodalisme, in: Annales ESC 37/1 (1982) $164 \mathrm{ff}$. 
Indem wir die hier gemachten Beobachtungen im Auge behalten, gehen wir jetzt zu den polnischen Angelegenheiten über. Es war die Monarchie der ersten Piasten, die den folgenschweren Versuch unternahm, die „Distribution der gesellschaftlichen Rollen“ mit Hilfe eines ganzen Netzes funktionaler Gruppenrechte durchzuführen. Diese Monarchie bemühte sich, vom Standpunkt ihrer eigenen Bedürfnisse aus die Aufgaben und gleichzeitig die besondere Position sowohl der sie unterstützenden Krieger als auch der Geistlichen und der ganzen Plejade der Freien, die zu den verschiedensten Leistungen verpflichtet waren, festzulegen. Es wurden auch nicht die Unfreien und die fremden Ankömmlinge - die Gäste - vergessen. Wenn wir auch nicht im einzelnen untersuchen wollen, wie das erwähnte System in der Praxis funktionierte, so bleibt doch festzuhalten, daß der Sinn seines Bestehens durch die Entwicklung der großen geistlichen und weltlichen Grundherrschaften untergraben wurde, wozu die Erlangung von Immunitäten sowie die schnelle Umwandlung der Landwirtschaft auf der Basis des Zinssystems im 13. Jahrhundert wesentlich beitrugen. Die Privilegien der Teilfürsten taten das ihre, um die Lage der Geistlichkeit innerhalb der polnischen Kirchenprovinz zu vereinheitlichen, die Rechte und Pflichten des Rittertums in den einzelnen Ländern anzugleichen und schließlich den rechtlichen Rahmen für eine Autonomie zu schaffen, die in zunehmendem Maße die städtischen Zentren betraf.

Eine weitere Vereinheitlichung der Position der einzelnen gesellschaftlichen Gruppen und Schichten war im Rahmen des 1320 wiedervereinten polnischen Königreiches eine Notwendigkeit ${ }^{7}$. In der Praxis umfaßte dieser Prozeß jedoch nur die beiden am stärksten privilegierten Gruppen - die Geistlichkeit sowie den die Herren und die Ritterschaft vereinigenden Stand der Schlachta.

Der rechtliche Rahmen dieses Standes bestimmte die weiteren Generalprivilegien, die von fremden Herrschern ausgestellt wurden, die nach dem Aussterben der nationalen Dynastie - den Anjou und den Jagiellonen - auf den polnischen Thron berufen wurden ${ }^{8}$. Was die von unten her verlaufenden Integrationsprozesse sowie das gemeinschaftliche Standes- und Korporationsbewußtsein anbelangt, so zeugt von deren bzw. dessen Festigung das Auftauchen der Überzeugung (und zwar sowohl innerhalb als auch außerhalb der analysierten Gruppe), daß die privilegierte Stellung in der Gesellschaft schon nicht mehr einzig das Ergebnis der Ausübung würdevoller Funktionen

7 Viele Aspekte dieses Prozesses auf vergleichbarem Hintergrunde bei: Karol Górski, Communitas, Princeps, Corona Regni. Studia selecta, Roczniki Towarzystwa Naukowego w Toruniu, Bd. 78, H. 1 (Warszawa 1976): Stanistaw Russocki, Monarchie et états dans le centre-est de L'Europe aux temps de Charles IV, in: Medzinárodní Vêdecká Konference - Doba Karla IV ... Materialy ze Sekce dêjin státu a práva, (Universita Karlova, Praha 1981) 51 ff.; idem, Les structures politiques dans l'Europe des Jagellons, in: APH XXXIX (1979) $101 \mathrm{ff}$., idem, The Parliamentary Systems in 15th-Century Central Europe, in: Poland at the 14th International Congress of Historical Sciences in San Francisco (Wrocław 1975) $7 \mathrm{ff}$.

${ }^{8}$ Cf. Antoni Gasiorowski, Research into Medieval Polish Nobility, in: The Polish Nobility in the Middle Ages. Anthology, ed. A. Gąsiorowski (Polish Historical Library 5, Wrocław 1984) 7 ff.; im folgenden zitiert: Gasiorowski, The Polish Nobility; Karol Buczek, The Knight Law and the Emergence of the Nobility Estate in Poland, in: ut supra $87 \mathrm{ff}$.; J. Bieniak, Knight Clans in Medieval Poland, in: ut supra, 123 ff.; Antoni Gasiorowski, Rotation of the Elite of Power in Medieval Poland, in: ut supra, $207 \mathrm{ff}$; Stanistaw Russocki, The Origins of Estate Consciousness of the Nobility of Central Europe, in: APH XLVI (1982) $31 \mathrm{ff}$. 
oder der entsprechenden Ausrüstung des berittenen Kriegers war, sondern von der edlen Geburt sowohl väterlicher- als auch mütterlicherseits abhing. Ausdruck dieses Bewußtseins wurden die seit Ende des 14. Jahrhunderts immer zahlreicheren Gerichtsprozesse über die Beanstandung der Zugehörigkeit zur Schlachta. Es sei am Rande bemerkt, daß eben diese Prozesse dank der Bestechlichkeit der Zeugen den begüterten Angehörigen des Bürgerstandes die Möglichkeit eröffneten, in den höchstprivilegierten Stand aufzusteigen.

Es wurde schon betont, daß trotz aller Bemühungen der hohen Herren - insbesondere der kleinpolnischen -, sich als besonderer Stand zu konstituieren (diese Bestrebungen entwickelten sich aus solchen objektiven Gegebenheiten wie dem Monopol der wohlhabenderen Geschlechter im Bereich der Land- und Kronenämter oder den Eheschließungen innerhalb der Gruppe) ${ }^{9}$, sich ein einziger Stand der Hochgeborenen ausbildete, was als spezifisches Merkmal der polnischen Bedingungen anzusehen ist. Nach dem Grundsatz formaler Gleichheit vereinte dieser Stand sowohl die mächtigen als auch die mittelbegüterten und sogar die kleinen, mit eigener Hand die Scholle bearbeitenden Ritter. Als faktisch einziger politischer Stand begann seit der Mitte des 15. Jahrhunderts die Schlachta die Funktion eines Fürsprechers der Interessen der gesamten Gesellschaft sowie des Staates und der Leitung desselben gemäß den eigenen Wünschen zu monopolisieren. Im Lauf des 16. Jahrhunderts begann der Stand der Schlachta sich sozusagen mit der Nation der Adelsrepublik zu identifizieren.

Viel schwächer zeichneten sich in Polen die Umrisse der Standesstruktur des Bürgertums ab. Sogar in den Kreisen des königlichen Hofes sprach man von den Bewohnern unserer Städte, nicht aber von unseren Bürgern. Obwohl die Städte tatsächlich dem allgemeinen System der staatlichen Verwaltung nicht unterlagen und eigene Gerichte und Rechte besaßen, gingen die Bande der Standessolidarität und des sie begleitenden Bewußtseins nicht über die Grenzen der einzelnen Zentren hinaus. Vergeblich waren die Bemühungen Krakaus, die größeren Städte in einer Art Gruppe mit gemeinsamen Interessen zu verbinden. Die Teilnahme der Städte am politischen Leben des Staates war sehr bescheiden. In einigen internationalen Verträgen traten sie als Mitbürgen auf; nach dem Tode von Ludwig von Anjou wurden sie zu lokalen konföderativen Verbänden zugelassen, deren Aufgabe es war, Ordnung und Ruhe zu bewahren; in Sachen der reparatio monetae wurden Bürger - aber nur diejenigen von Krakau - einbezogen. Gegen Mitte des 15. Jahrhunderts wurden die Städte der wichtigen Berechtigung beraubt, die analogen Institutionen in einem Großteil Europas längst zustand - nämlich der Zustimmung zur Erhebung außergewöhnlicher Steuern.

Dies führte in der Folge zur Eliminierung der Bürger aus den polnischen Ständeversammlungen. Wie anders sah die Situation der Städte in dem nach 1466 mit der Polnischen Krone vereinten und eine politische Einheit bildenden Königlichen Preußen aus ${ }^{10}$ ! Bis heute sind die Ursachen für den desolaten Zustand städtischer Reprä-

9 Cf. H. Litwin, The Polish Magnates 1454-1648. The Scaping of an Estate, in: APH LIII (1986) $63 \mathrm{ff}$.

${ }^{10}$ Cf. Marian Biskup, Die Rolle der Städte in der Ständevertretung des Königreiches Polen, einschließlich des Ordensstaates Preußen im 14./15. Jahrhundert, in: Städte und Ständestaat ..., hrsg. v. Bernbard Töpfer (Forschungen zur Mittelalterlichen Geschichte 26, Berlin 1980) 163 ff.; 
sentation nicht völlig geklärt. Nur einige wenige Gründe seien angeführt: die allgemeine, relative Schwäche der polnischen Städte, ihre Unfähigkeit, der Monarchie entsprechende materielle Hilfe zu gewähren; die Unterbewertung der potentiellen Bedeutung der Städte im politischen Leben durch die Vertreter der Jagiellonendynastie; die Unfähigkeit, den unaufhörlich wachsenden Standesegoismus der Schlachta zu hemmen.

Bei der bis über das 16. Jahrhundert hinaus im allgemeinen günstigen Wirtschaftskonjunktur fehlte den Städten die Motivation, sich von den anderen Ständen abzugrenzen. Die Kontakte mit der Schlachta schienen die privaten und Gruppeninteressen zu garantieren ${ }^{11}$; darüberhinaus eröffneten sie den Mitgliedern der bürgerlichen Oberschicht die Möglichkeit, in den Stand der „Hochwohlgeborenen“ $\mathrm{zu}$ avancieren. Der Zustrom der Bauern in die Städte, die ja in bedeutendem Maße ihren landwirtschaftlichen Charakter bewahrten, bedeutete keinerlei Konkurrenz.

Was das Bauerntum betrifft - hier sei der theoretische Streit, ob diesem überhaupt die Bezeichnung "Stand“ zusteht, übergangen -, so muß festgestellt werden, daß es „als solches“ sowohl von der Monarchie des 14. bis 15. Jahrhunderts als auch von den damaligen Kreisen der Intellektuellen behandelt wurde. Es wurde von den die Bauern belastenden Pflichten gesprochen, es wurden deren Rechte reguliert. Es scheint, daß die Bauern überhaupt keine Möglichkeit hatten, ein allgemeines Standesbewußtsein zu entwickeln, da die zwischenmenschlichen Kontakte infolge der schwachen Entwicklung der Kommunikationssysteme kaum möglich waren; außerdem waren sie in Herrschaftsbereichen verschiedener Organisationsformen (königliche, kirchliche, große, mittlere und kleine Landbesitzungen) verstreut. Die Grenzen des Standesbewußtseins der Bauern wurden gewiß durch eigene Erfahrungen geprägt, die bei den jeweils herrschenden Herren oder deren Nachbarn gemacht wurden. Eine Entwicklung von eigenem Standesbewußtsein wurde zusätzlich durch die Tatsache erschwert, daß eben diese noch in den Anfängen der Zinswirtschaft begann - als die ländliche Bevölkerung sich in zwei grundsätzliche Gruppen teilte. Die eine bestand aus den persönlichen Freien, die den staatlichen Gerichten unterstanden, sich auf dem Grund und Boden der Herren infolge eines individuellen Abkommens, das sozusagen eine zeitlich unbegrenzte Pacht darstellte, ansiedelten, aber jeden Moment wieder weiterziehen konnten, falls sie die übernommenen Verpflichtungen erfüllt hatten. Die andere Gruppe umfaßte Siedler in Dörfern, die aufgrund des Deutschen Rechtes angelegt worden waren, das zwar ein besseres Recht auf das Land gewährte, aber das Verlassen des Dorfes erschwerte; diese Siedler unterstanden zusätzlich - wenigstens teilweise der gerichtlichen Immunität und befanden sich demgemäß in einer Situation der grundherrschaftlichen Abhängigkeit. Diese Unterschiede begannen sich erst gegen

\section{Fortsetzung Fußnote von Seite 173}

M. Ludwig, Besteuerung und Verpfändung Königlicher Städte im spätmittelalterlichen Polen (Gießener Abhandlungen zur Agrar- und Wirtschaftsforschung des europäischen Ostens 126, Berlin 1984).

${ }^{11}$ Cf. Jacek Wiesiotonski, The Nobility in Town. Movements and Migration of the Nobility between the Village and Town in Poland during the 15th Century, in: Gasiorouski, The Polish Nobility, $255 \mathrm{ff}$. 
Ende des 15. Jahrhunderts zu verwischen, und zwar im Zusammenhang mit der Ausbreitung der sog. sekundären Leibeigenschaft ${ }^{12}$.

Hier fügen wir noch folgende Bemerkung hinzu, und zwar in Verbindung mit den Bemerkungen zum Thema Eigenschaften der Ständegesellschaft, innerhalb derer sich von beiden Seiten - von oben und von unten - die Strukturierungsprozesse vollzogen: Außer der klassischen vierteiligen Gliederung der polnischen Gesellschaft des 14. Jahrhunderts in die Geistlichkeit, die Schlachta, das Bürgertum und die Bauern, bestanden Tendenzen einer Anreicherung dieser Teilung. Über eine von ihnen, die höchstwahrscheinlich von den kleinpolnischen Herren ausgegangen war, die sich als besonderen Stand konstituieren wollten, haben wir schon gesprochen. Die andere, die gewiß vom Staat selbst initiiert worden war, betraf die Schulzen, die Vorsteher der Dorfgemeinden, die gemäß den Grundsätzen des ius theutonicum organisiert waren. Sie stammten vorwiegend aus der Schlachta oder dem Bürgertum und verstanden es, in ihren Händen ziemlich große, einige Hufen umfassende Wirtschaften in Form von Vorwerken zu organisieren. Die Art und Weise, wie diese Personen ihren Wehrdienst - ähnlich wie die Ritter - absolvierten, sowie die Tatsache, daß sie der Jurisdiktion der Gerichte von Standesgleichen unterstanden, bildeten ein ausreichendes Motiv, um die Schulzen in einen Stand zu verwandeln. Diese Initiative schlug jedoch fehl, da die Schlachta sich ihr energisch widersetzte, schaute sie doch voller Neid auf die Landgüter der Schulzen und auf deren Stellung innerhalb eines Dorfes ${ }^{13}$.

Die hier dargelegte, zwangsweise sehr summarische Übersicht der Probleme scheint zur Formulierung folgender Konklusionen zu berechtigen:

1. Seit Beginn des 13. Jahrhunderts wandelte sich die polnische Gesellschaft in eine Ständegesellschaft, das heißt, die Positionen der einzelnen großen gesellschaftlichen Gruppen wurden durch die Funktionen (Rollen) determiniert, die jene im Rahmen der staatlichen Organisation ausübten, sowie durch die ihnen in Konsequenz davon von den Herrschern zugesprochenen Rechte und Privilegien.

2. Eine vollkommene Integration und - gleichzeitig - das Bewußtsein, einem korporativ organisierten Stand anzugehören, erlangten nur der geistliche Stand und der Stand der Schlachta. Der letztere war seit der Mitte des 15. Jahrhunderts faktisch der einzige politische Stand des Königreiches, er verkörperte dessen gesamte Gesellschaft und konkurrierte mit der Monarchie im Rahmen des ebenfalls in Polen funktionierenden dualistischen Machtsystems.

3. Die Bestrebungen der Schlachta, ihre dominierende, privilegierte Position in allen Bereichen des wirtschaftlichen, gesellschaftlichen und politischen Lebens zu behalten, begrenzten und hemmten die Integrationsprozesse der anderen Stände, vor allem des Bürgertums. Sodann nahm seit Beginn des 16. Jahrhunderts die Adels-

12 Cf. Stanistaw Russocki, Ritterliches Gutseigentum mit und ohne Grundherrschaft im spätmittelalterlichen Polen, besonders in Masovien, in: Grundherrschaft im Späten Mittelalter I., hrsg. von Hans Patze (Vorträge und Forschungen ... XXVII, 1, Sigmaringen 1983) $577 \mathrm{ff}$; Stanistau Russocki, Les "groupes d'intérêt" dans la sociéte féodale polonaise, APH XIV (1966) $75 \mathrm{ff}$.

13 Cf. L. Eysiak, Wlasność soltysia (wójtowska) w Małopolsce do końca XVI wieku (De dominio scultetorum in Polonia Minori usque ad finem saec. XVI) (Zeszyty Naukowe Uniwersytetu Jagiellonskiego XCIV. Prace prawnicze 15, Kraków 1964). 
republik ihre Anstrengungen wieder auf, eine rigorose standesmäßige Strukturierung der Gesellschaft zu schaffen. An die Stelle der Gemeinschaft von Gruppenrechten traten immer häufiger den Bürgern und Bauern auferlegte Begrenzungen und Verbote.

4. In Anbetracht des oben gesagten und auch mit der Absicht, eine gemeinsame Sprache mit den Forschern anderer, vor allem nachbarlicher Staaten aufrechtzuerhalten, sind wir dafür, dem Polen des ausgehenden Mittelalters nicht das Attribut der Standesmäßigkeit abzuerkennen, sondern uns für weitere Erforschungen seiner Besonderheiten einzusetzen. Wie vielversprechend solche Forschungen sein können, beweisen die neuesten Studien von Henryk Samsonowicz.

Übersetzt von Beatrix Hirschenberg. 